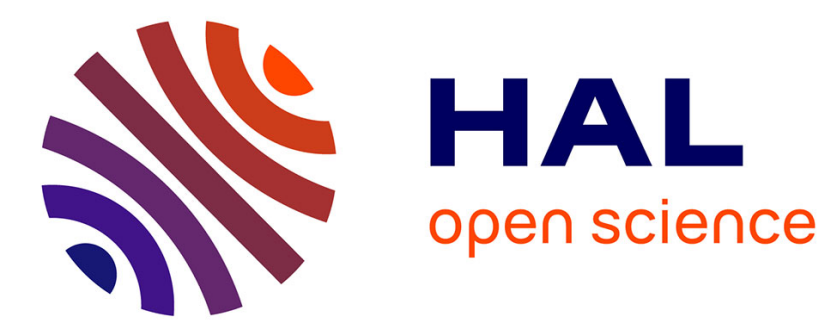

\title{
Communauté et société. Collingwood contre les sociologues
}

\author{
Laurent Jaffro
}

\section{To cite this version:}

Laurent Jaffro. Communauté et société. Collingwood contre les sociologues. Dialogue: Canadian Philosophical Review / Revue canadienne de philosophie, 2008, 47 (2), pp.253-271. halshs-00351723

\section{HAL Id: halshs-00351723 \\ https://shs.hal.science/halshs-00351723}

Submitted on 21 Feb 2009

HAL is a multi-disciplinary open access archive for the deposit and dissemination of scientific research documents, whether they are published or not. The documents may come from teaching and research institutions in France or abroad, or from public or private research centers.
L'archive ouverte pluridisciplinaire HAL, est destinée au dépôt et à la diffusion de documents scientifiques de niveau recherche, publiés ou non, émanant des établissements d'enseignement et de recherche français ou étrangers, des laboratoires publics ou privés. 
Brouillon de "Communauté et société. Collingwood contre les sociologues», Dialogue Canadian Philosqphical Review X LVII, 2 (2008), p. 253-271.

\section{Communauté et société. Collingwood contre les sociologues}

Laurent Jaffro Université Blaise Pascal, Clermont-Ferrand

\section{Laurent.Jaffro@univ-bpclermont.fr}

Dans le Nouveau Léviathan (1942) ${ }^{1}$, le lecteur découvre, parmi bien d'autres choses, les éléments d'une philosophie sociale, c'est-à-dire une théorie qui examine la notion de société en procédant de manière conceptuelle et non pas empirique. Comme la plupart des thèses que défend Collingwood, cette philosophie sociale a une dimension très polémique et se présente explicitement comme la contradiction des vues qui prédominent dans la sociologie depuis le XIX ${ }^{\mathrm{e}}$ siècle. Cette étude reconstitue le contexte polémique dans lequel s'inscrit la distinction que propose Collingwood entre la communauté, entendue comme une communauté non sociale, régie par des rapports de contrainte et des motivations seulement affectives, et la société, c'est-à-dire la communauté proprement sociale, régie par des rapports de volonté et des motivations aussi rationnelles. Des distinctions semblables ont été proposées par des sociologues avant que Collingwood ne les reformule et elles resteront, sous une forme ou sous une autre, importantes dans la sociologie du $\mathrm{Xx}^{\mathrm{e}}$ siècle. Ce qui est d'abord original sous la plume de Collingwood, c'est moins la caractérisation des termes entre lesquels la distinction opère que la manière de la concevoir.

L'initiative de Collingwood, en effet, consiste à substituer à la manière dont la sociologie distingue entre communauté et société une autre manière de distinguer. Pour aller à l'essentiel, la distinction que pratique en l'espèce Collingwood est exactement celle qu'il a théorisée ailleurs sous le nom d'échelle des formes et qui, selon lui, s'applique aux concepts philosophiques. Le Nouveau Léviathan pratique de manière implicite une conception de la distinction qui n'est explicitée que dans d'autres textes, spécialement dans l'Essay on Philosophical Method. La différence entre communauté et société n'est ni simplement de nature, ni simplement de degré, mais est une différence qui relève à la fois de l'un et de l'autre 2 . Ce que Collingwood dit de la distinction entre communauté et société pourrait être résumé en ces quelques mots : la communauté (non sociale) et la société (ou communauté sociale) sont deux espèces du genre communauté, qui se distinguent l'une de l'autre selon le type de différence dont Collingwood dit qu'elle convient aux concepts philosophiques, par opposition aux concepts des sciences empiriques ou des mathématiques, c'est-à-dire de telle façon que, simultanément,

1) la communauté non sociale est une réalisation très imparfaite de l'essence de ce qu'est la communauté, tandis que la communauté sociale se place plus haut sur l'échelle de la réalisation de l'essence;

${ }^{1}$ Toutes les citations au Nouveau Léviathan sont indiquées dans cet article par l'abréviation $N L$ suivie du numéro de paragraphe.

2 Voir l'introduction de David Boucher à Collingwood, 1989 (p. 34-35). 
2) la communauté sociale est aussi toujours non sociale à certains égards et, inversement, la communauté non sociale est déjà une communauté et, à cet égard, amorce l'espèce supérieure.

En un sens, tout est dit. On pourrait même penser que si la façon dont Collingwood conçoit la différence entre communauté et société n'est pas limpide, cela tient en réalité directement à la conception qu'il a de la différence entre des concepts philosophiques, et pas spécialement aux concepts de communauté et de société auxquels il applique cette conception de la différence. C'est pourquoi, afin d'aller un peu plus loin, je vais laisser provisoirement de côté l'aspect sous lequel nous avons affaire à un exemple de la différence qui est à l'œuvre dans une échelle des formes. Je considérerai la distinction en elle-même et ferai comme si Collingwood avait participé aux discussions de ce qu'on dénommait la sociologie pure et qui consistait en l'élucidation des concepts fondamentaux. À cette fin, j'aborderai les notions de communauté et de société en tant qu'elles relèvent des catégories de la sociologie pure.

Le premier objectif, historique, est de reconstituer le contexte polémique. Il me semble que Collingwood vise directement les catégories de la sociologie pure et en particulier la manière dont Ferdinand Tönnies (1855-1936) avait fait cette distinction (en allemand) en 1887, dans son ouvrage Gemeinschaft und Gesellschaft (Tönnies, 1944) ${ }^{3}$. Collingwood ne mentionne pas Tönnies dans le Nouveau Léviathan, et je ne connais pas de passage où il discute Tönnies. Il y a cependant plusieurs motifs de conjecturer que Tönnies ou du moins une famille de pensée qui est bien illustrée par lui est la cible de Collingwood.

Le second objectif est plus général : situer la philosophie sociale de Collingwood par rapport aux principes et aux méthodes de la philosophie sociale des sociologues. Je montrerai que, bien que Durkheim ait pu manifester son désaccord avec la manière dont Tönnies distinguait communauté et société, Collingwood n'est pas plus d'accord avec Durkheim qu'avec Tönnies. Surtout, j'essaierai de montrer comment Collingwood se tient à distance des principes de la philosophie sociale des sociologues, alors même qu'il discute les concepts fondamentaux de la sociologie. J'essaierai de répondre à la question suivante : la dialectique de la communauté et de la société, dans le Nouveau Léviathan, constitue-t-elle aux yeux de Collingwood la base d'une nouvelle fondation des sciences sociales, auquel cas elle serait une sorte de sociologie pure alternative, ou bien s'agit-il au contraire pour Collingwood de contester dans son principe la sociologie?

${ }^{3}$ Le sous-titre de la première édition était «Traité sur le communisme et le socialisme en tant que formes empiriques de la culture». Le sous-titre de l'édition suivante, vingt-cinq ans plus tard, est «Catégories fondamentales de la sociologie pure». Ce changement traduit un revirement de Tönnies à l'égard du socialisme politique et l'adoption d'une position moins pessimiste à propos de la modernité (Mitzman, 1971). 


\section{Community/Society versus Gemeinschaft/Gesellschaft}

Selon Tönnies, il existe un concept de la communauté (Gemeinschaft $\left.{ }^{4}\right)$ qui s'applique univoquement aux animaux et aux hommes, parce que «la vie collective est une donnée de la nature» (Tönnies, 1944, p. 25). Au contraire, selon Collingwood, il y a un sens strict de «société» qui interdit qu'on l'étende aux animaux et aux autres êtres que l'homme, et comme toute communauté a un noyau de société, on doit considérer que l'assimilation des communautés humaines même les moins sociales et les plus rudimentaires à des sociétés ou communautés purement naturelles (animales) est abusive. Le chapitre 19 du Nouveau Léviathan s'efforce de restituer au terme «société» son sens propre d'association volontaire, contre l'usage métaphorique, biologisant, qui s'est répandu au XIX ${ }^{\mathrm{e}}$ siècle et qui trouve un exemple dans Tönnies comme en d'autres auteurs qui ont en quelque sorte appliqué au développement des sociétés une conception évolutionniste en un sens assez large du terme, mieux illustré peut-être par Herbert Spencer que par Charles Darwin.

Pour Tönnies, la communauté (Gemeinschaft) qui est une organisation fondée sur le sang, le lieu, l'instinct, est essentiellement distincte de la société, Gesellschaft, qui, elle, est une association volontaire et repose sur un contrat, c'est-à-dire une décision librement consentie. Tönnies s'inspire ici nettement des thèses de l'historien anglais du droit Henry Sumner Maine (1822-1888), notamment de sa distinction entre les communautés anciennes régies par un statut et les communautés modernes qui sont réglées par un contrat 5 . On trouve une distinction semblable chez l'anthropologue américain Lewis Henry Morgan (1818-1881) qui est une autre source de Tönnies ${ }^{6}$. Il est important de souligner que les sources de Gemeinschaft und Gesellschaft ne sont pas seulement allemandes, mais aussi anglosaxonnes, faute de quoi on risquerait de tomber dans des simplifications qui favorisent une germanophobie d'un genre collingwoodien. Selon Tönnies, la Gemeinschaft est une unité dans la pluralité, une unité organique, et la Gesellschaft est la pluralité dans l'unité, un agrégat mécanique. Cette différence de nature entre communauté et société s'accompagne d'une priorité de la communauté sur la société qui est non seulement une priorité logique et chronologique, mais une priorité en dignité et en valeur : «Communauté est vieux, société est nouveau comme chose et comme nom» (Tönnies, 1944, p. 4). Comme on sait, c'est toujours mieux avant. La Gesellschaft est une forme décadente de la vie collective, liée à la corruption du lien social qu'entraîne le développement

${ }^{4}$ Le terme community, en anglais, suggère une communauté plus ouverte, à tel point que le traducteur anglais, Charles Loomis avait conservé les termes allemands (Tönnies, 1940). Voir Bond, 2002, p. 106.

${ }^{5}$ Maine, 1861, cité par Tönnies, 1944, p. 176-177. Sur Maine, voir Orenstein, 1968.

${ }^{6}$ Morgan, 1877. Pour l'anecdote, le sous-titre de l'ouvrage anticipe celui du Nouveau Léviathan. Entre autres chose, Morgan est aussi l'auteur d'une étude sur The American Beaver and His Works (1868) qui voit entre les castors et les êtres humains de simples différences de degré. Ce genre de naturalisme correspond exactement à ce qui est l'objet constant de l'ironie de Collingwood. 
économique urbain; la campagne moderne, qui conserve les modes de vie ancestraux, offre encore des exemples de communauté. L'authenticité, la naturalité, la vitalité sont du côté de la communauté villageoise. Tönnies fonde ainsi son opposition de la communauté et de la société sur la considération des effets désocialisants de la vie moderne, spécialement urbaine. Il s'inscrit à cet égard dans les premiers développements de la science de l'homme qui, depuis les Écossais, particulièrement Adam Ferguson, jusqu'à Marx (Tönnies, 1944, p. 53-54), avait dénoncé les dégâts qu'entraînent dans la société les formes extrêmes que revêt la division du travail. Cette sociologie, très normative en dépit des protestations de Tönnies, a été, au moins dans ses premières formulations, le support d'une sorte de communisme politique qui espérait que la Gesellschaft s'effondrerait sur elle-même. Au terme de sa carrière, Tönnies réfléchissait encore aux formes associatives susceptibles de développer les traces de la communauté archaïque dans la société moderne.

Une différence majeure entre Collingwood et les sociologues qui ont proposé ce genre de distinction est, d'abord, que Collingwood reprend la distinction entre communauté et société, en lui donnant comme base la considération non pas du rapport économique, mais du rapport juridique. Collingwood voit dans la société le rapport entre des volontés qui passent des contrats afin de coopérer, et non pas le rapport d'échange entre des individus uniquement préoccupés d'eux-mêmes. Si l'on substitue ainsi la perspective juridique à la perspective économique, on peut alors prétendre que la société comme chose et comme nom n'est pas moins ancienne que la communauté. Selon le Nouveau Léviathan, elle trouve à la fois sa première réalisation et sa première conceptualisation dans le droit romain. C'est une façon, il me semble, de contester l'interprétation que donnait Henry Sumner Maine du droit romain et d'insister sur la liberté là où Maine voyait essentiellement de la contrainte. À ce propos, Collingwood souligne avec un certain embarras que

les juristes romains s'intéressaient à l'association surtout à cause des questions économiques qu'elle implique parfois. Mais en soit elle n'implique nullement ces questions, et en fait les spécialistes du droit romain nous ont laissé une théorie de l'association qui reste valable, avec des modifications très légères et évidentes, quand nulle question économique ne se pose. ( $N L$ 20.81)

En détachant le concept de la société des considérations économiques pour lui redonner son sens juridique d'association volontaire, Collingwood tire simplement un trait sur toute l'histoire de la discussion autour de la notion de civil society ou de bürgerliche Gesellschaft qui avait couru de Ferguson à Hegel et Marx : cette discussion réduisait la société et plus généralement les rapports contractuels à l'échange marchand7 et, solidairement, à la division du travail et à l'égoïsme des acteurs

${ }^{7}$ Le contrat «est la résultante de deux volontés divergentes qui, en un point, se coupent. Il dure jusqu'à l'achèvement de l'échange» (Tönnies, 1944, p. 45). Comme les hommes de la société sont séparés et non déterminés par une unité préalable et plus forte, leur lien consiste seulement dans l'échange : à 
qui en sont l'envers. La société civile était alors vue comme le lieu même de la désocialisation puisque les individus n'y poursuivent que leurs intérêts personnels. L'appréciation du lien contractuel est diamétralement opposée. Là où Collingwood voit une communauté de volonté, Tönnies, à la suite de Ferguson, de Hegel, de Marx bien sûr, considère l'atomisation de la société qui ne laisse subsister du lien que le rapport économique entre les individus : «Tandis que, dans la communauté, ils restent liés malgré toute séparation, ils sont, dans la société, séparés malgré toute liaison» (Tönnies, 1944, p. 39). Une autre façon de résumer la position de Collingwood consiste à dire que la distinction entre communauté et société est mieux faite en anglais (c'est-à-dire en latin) qu'en allemand, parce que, selon lui, le terme society renvoie au rapport volontaire et même libre entre des associés ou des sociétaires (ibid., 19.94, p. 161), tandis que le champ sémantique du terme Gesellschaft est dissemblable: la Gesellschaft est une collaboration, un partenariat, une simple compagnie au sens commercial du terme.

Si nous revenons maintenant vers l'autre pôle de la distinction, à savoir la notion de communauté (non sociale), la question cruciale pour la caractériser est dans la détermination de ce qu'est la famille, puisqu'on peut s'accorder sur le fait que le cœur de cette communauté est dans les rapports familiaux. Tönnies trouve dans la famille et en particulier dans l'instinct sexuel le noyau de la Gemeinschaft. Le modèle de la communauté est le chez soi, en comparaison duquel la société est toujours le milieu dans lequel on doit entrer comme un étranger, où l'on ne connaît personne (le monde, le grand monde, spécialement les métropoles), dont on doit se méfier, où l'on peut se perdre. Tönnies ne fait pas de différence entre la situation des enfants par rapport à la famille et la situation des époux qui constituent le noyau familial. Dans les deux cas, le lien est communautaire, c'est-à-dire repose sur un rapport entre des volontés essentielles (Wesenwille) ou (selon la traduction française ${ }^{8}$ ) «volontés organiques»: ce qui signifie que le rapport est profondément involontaire, parce que sentimental, affectif, libidinal. «On se trouve en communauté avec les siens depuis la naissance, lié à eux dans le bien comme dans le mal» (Tönnies, 1944, p. 4).

L'usage de la notion de volonté par Tönnies appelle des explications. Personne n'a choisi ses parents. Un enfant ne choisit pas le lien familial dans lequel il se trouve pris. Ce rapport est involontaire au sens où il n'a pas été décidé par l'intéressé. Pour que Tönnies puisse rapporter le lien de l'enfant aux parents en termes de volonté, il faut qu'il entende par là quelque chose de tout différent du choix, de la décision. Il entend par Wesenwille une détermination du désir ou de l'affect. De plus, pour Tönnies, le rapport entre les parents n'est pas moins de communauté que le rapport de l'enfant avec les parents. Il joue sur les divers sens des termes Gemeinschaft et Gesellschaft en allemand, sur le

une situation où chacun est pour soi, correspond un lien qui est simple commerce de services mutuels (Tönnies, 1944, p. 39).

8 Le commentaire anglophone parle plus volontiers de «essential will», mais Loomis emploie également le terme «organic» dans sa traduction. 
fait surtout que Gesellschaft, suppose une collaboration distante, une étrangeté réciproque qui sied mal au lien conjugal: «les fiancés savent qu'ils entrent dans le mariage comme dans une pleine communauté de vie (communio totius vitae). Une société (Gesellschaft) de vie est une expression contradictoire dans les termes» (ibid.). Un mariage n'est pas une petite entreprise. Mais on pourrait objecter à Tönnies que ce qui compte, ce n'est pas de savoir si les époux entrent dans une communauté de vie, mais de savoir de quelle manière ils y sont entrés : si c'est par un contrat ou bien à la façon dont les enfants sont entrés dans cette communauté, c'est-à-dire, de leur point de vue, involontairement. En d'autres termes, dans la structure familiale, il y a un élément involontaire (au sens de non décidé par l'intéressé) qui tient à la naissance et au sang, et un élément volontaire (pas toujours, mais l'essentiel est, comme l'a bien vu Collingwood, qu'il puisse être volontaire) qui tient au contrat qui lie les époux. Pour démontrer l'ancienneté et la primitivité de la communauté, son antériorité par rapport à toute société, Tönnies amalgame l'élément volontaire avec l'élément involontaire, contrairement à ce qu'exigerait une description correcte de la famille, car en aucun cas le lien entre époux n'est assimilable à un lien du sang ou de la naissance. C'est seulement au prix de cette distorsion que Tönnies peut faire de la famille la communauté fondamentale et l'origine de toute Genossenschaft.

C'est seulement dans les liens du sang que se présente de la façon la plus immédiate l'unité et, par suite, la possibilité d'une communauté des volontés humaines, c'est en second lieu dans le rapprochement spatial, et aussi enfin, pour les hommes, dans le rapprochement spirituel. C'est par conséquent dans cette classification qu'il faut rechercher les racines de tous les rapports (associations) (Tönnies, 1944, p. 21).

À rebours, selon Collingwood, la famille dans son noyau n'est pas une communauté non sociale mais est, partiellement, une société. Il y a une partie qui se gouverne elle-même, et une partie, la pouponnière, qui est gouvernée : «Une famille est ce que j'appelle une communauté mixte, c'est-à-dire qu'une partie en est une société, l'autre partie, que j'appelle le groupe des enfants (nursery), est une communauté non sociale» (NL 22.1). De plus, le noyau social est présent dans la plupart des communautés, sinon dans toutes (ibid., 22.2). Quel est le noyau social de la famille? C'est le contrat social qu'est le mariage (ibid., 22.34 et 22.40). Ici Collingwood ne prétend pas donner une théorie complète ni une description exhaustive des formes de vie familiales, mais seulement prendre un cas simple de communauté pour montrer qu'elle est mixte. Cela lui permet de dégager ce qu'il appelle la structure de la famille normale :

C'est une communauté qui dans l'ensemble est non sociale, bien qu'elle contienne un noyau social. La volonté libre n'est pas indispensable pour en être membre. Ce n'est pas par la volonté libre du nourrisson qu'il devient, comme certainement il le devient, membre de la famille. J'ai appelé les parents le noyau social de cette 
communauté. Ceci implique que le mariage est un contrat social entre un homme et une femme (ibid., 23.3 et 23.31, p. 192-193).

Quant à la partie non sociale de la famille, elle tient au fait qu' «un homme naît masse rouge et ridée de chair sans aucune volonté à soi, absolument à la merci des parents par la coalition de qui il a été amené à l'existence» (ibid., 23.96, p. 203).

Collingwood admet que dans «les plus anciens documents du droit romain» on découvre «une société où cette croyance n'avait pas cours». Au temps que décrivent les «douze tables», «une femme devait toujours être dans la manus de quelqu'un» et n'avait pas la capacité de conclure un contrat. Dans ce passage, il me semble évident que Collingwood est en train de paraphraser ironiquement Lewis Henry Morgan et Henry Sumner Maine, car il s'agit exactement de l'objet de leurs réflexions respectives sur le droit archaïque. Tout ce qu'on peut conclure de telles observations, selon Collingwood, c'est que

1) l'éducation des femmes n'était pas du tout la même à cette époque que dans l'Europe moderne;

2) cela n'empêche pas que le droit romain fournit le cadre dans lequel on peut penser le noyau social de la famille moderne;

3) même dans la société moderne un mariage peut commencer par être «un peu moins qu'un contrat» et devenir ensuite un peu plus un contrat (ibid., 23.47).

Le meilleur argument contre le théoricien de la patria potestas qu'incarnait Henry Maine et l'admirateur des castors qu'était Lewis Henry Morgan, c'est, pour Collingwood, qui ici se réjouit de choquer, l'argument de la technique contraceptive, qui a non seulement accru la liberté des contractants eu égard à la procréation, mais leur a révélé cette liberté, leur en a donné la conscience. «De nos jours, grâce à la connaissance des contraceptifs, il y a un grand nombre de personnes qui n'ont d'enfants, quand ils en ont, que par leur volonté libre» (ibid., 23.52, p. 196). La pratique de la contraception «a rendu les Européens modernes conscients d'une liberté dont jusque là ils n'étaient pas conscients - elle a accru leur liberté aussi bien que leur conscience de la liberté» (ibid., p. 202). Ce point est important car il permet à Collingwood d'invalider les théories biologisantes de la famille. La contraception permet au caractère volontaire de la paternité et de la maternité de se manifester. Grâce à elle, on peut décider d'avoir un enfant; c'est manifestement un objet de volonté. On ne saurait expliquer la paternité et la maternité par un «instinct parental», chose qui n'existe pas. Quand on fait un enfant sans l'avoir décidé «cela arrive non pas par l'opération de quelque "instinct parental" mais comme sous-produit de 1'“instinct" sexuel» (ibid., 23.86). Il existe un appétit sexuel (une tendance) qui devient un désir sexuel quand il est objet de conscience; mais la paternité ou la maternité doivent en être bien distingués.

Collingwood se lance dans une diatribe contre les ennemis de la contraception : «Il y a des gens dont le but est de limiter la liberté humaine [...]. Ils sont ennemis de la volonté libre» (ibid., 
23.88). Cette déclaration politique est aussi la position spéculative fondamentale de Collingwood: toute science de la société qui substitue l'instinct biologique ou toute chose de ce genre à la volonté libre contient un fond de barbarie. Je crois que Collingwood pouvait éprouver une joie spéciale à retourner contre l'anthropologie d'un Morgan la notion de barbarie. Au passage, il convient de noter que Collingwood semble souscrire à la thèse contestable selon laquelle plus il y a de situations de choix, mieux c'est pour ceux qui choisissent et plus ils sont libres. Il conviendrait sans doute, avec Amartya Sen, de bémoliser ce genre de considération en rappelant que pour caractériser le degré de liberté il convient également de tenir compte de la palette d'options disponibles et du degré d'importance (ou, à l'inverse, de trivialité) de ces options (Amartya Sen, 2000, p. 91-97).

Plusieurs motifs conduisent à estimer que la cible de Collingwood est bien constituée au minimum par une famille de pensée, celle de Maine, Morgan et Tönnies, et peut-être, mieux encore, par l'ouvrage de Tönnies lui-même, qui avait été abondamment réédité — il faut signaler que sa première traduction en anglais sous le titre de Fundamental Concepts of Sociology, par Charles Loomis, date de 19409, et non pas de 1957 puisqu'il s'agit alors de la seconde édition sous un autre titre, Community and Society. Le premier motif est simplement que Collingwood et Tönnies font l'un et l'autre référence à Hobbes. Bien que Collingwood, autant que je sache, ne cite pas Tönnies, il ne pouvait ignorer un aspect de son œuvre. L'édition par Tönnies, en anglais, des Elements of Law de Hobbes (1889) avait fait grand bruit et Collingwood aurait pu savoir que sa propre vision juridique d'un Hobbes individualiste et libéral10 s'opposait diamétralement à la vision qu'avait Tönnies d'un Hobbes absolutiste et, si l'on peut dire, communautariste11. La remarque du Nouveau Léviathan selon laquelle «le monde politique est un monde dialectique où les communautés non sociales (les communautés des hommes dans ce que Hobbes appelle l'état de nature) deviennent des sociétés» ( $N L$ 24.71) devient moins énigmatique quand on considère que Tönnies avait, à l'inverse, fini par assimiler le passage de l'état de nature à l'état politique à la supplantation de l'univers des individus égoïstes par une véritable Gemeinschaft. Collingwood prend l'exact contrepied de Tönnies sur ce point aussi. En outre, les remarques du Nouveau Léviathan sur la connivence entre Marx et les apologues de la communauté non sociale (ibid., 19.83, p. 160) sont moins surprenantes si l'on prend en considération l'usage que des auteurs comme Tönnies faisaient de Marx. Enfin, et c'est le point le plus important,

9 Cette traduction fait immédiatement l'objet d'une recension (Vance, 1941) qui justifie que Gemeinschaft et Gesellschaft restent en allemand sous la plume de Loomis en invoquant la notoriété des termes. Il faut signaler que dans une présentation synthétique de la sociologie de Tönnies, Louis Wirth avait utilisé les termes community et society (Wirth, 1926).

10 Rappelons que cette interprétation a la faveur de Collingwood, comme il le dit dans la préface du Nouveau Léviathan.

${ }^{11}$ Il faut signaler que la monographie de Tönnies, Hobbes Leben und Lehre (1896) n'a pas été traduite en anglais. 
comme Durkheim lui-même devait le souligner dans sa recension de l'ouvrage de Tönnies, une source d'inspiration de Gemeinschaft und Gesellschaft est Schopenhauer aux côtés de Marx, de Kant, de Sumner Maine et des évolutionnistes (Durkheim, 1975). La référence omniprésente à Hobbes, le rapprochement entre Marx et les sociologues biologisants, le fantôme de Schopenhauer ${ }^{12}$ et d'une volonté purement libidinale, tout ceci, dans le Nouveau Léviathan, constitue une sorte de portrait-robot dans lequel je suis tenté reconnaître Tönnies, mais aussi ses complices Maine et Morgan. C'est conjectural, mais il est difficile de se refuser à ce genre d'investigation si on veut pratiquer à l'endroit de Collingwood lui-même le type d'interrogation historique qu'il recommandait.

Le passage le plus significatif, à cet égard, est celui dans lequel Collingwood dénonce

la vieille propagande allemande menée contre la volonté en tant que telle, propagande dans laquelle le mot «volonté» est utilisé, peut-être pour la première fois par Schopenhauer, pour signifier quelque chose qui n'est nullement la volonté, mais seulement la passion ou le désir de quelque chose encore plus vil (ibid., 28.98, p. 255).

On ne saurait mieux décrire la manière toute schopenhauerienne dont Tönnies utilise le terme «Wille» dans son ouvrage. Pour Tönnies il y a deux types de volonté : «La volonté dans la mesure où elle contient la pensée, et la pensée dans la mesure où elle contient la volonté» (Tönnies, 1944, p. 81). Il y a une volonté qui instrumentalise la pensée, c'est la volonté naturelle ou essentielle (Wesenwille). Et une volonté dans laquelle le volitif est au service du cognitif et même du calcul, c'est la volonté artificielle, rationnelle, réfléchie: la volonté arbitraire (Willkür en 1887, Kürwille à partir de la deuxième édition). La volonté arbitraire est «un produit de la pensée», elle est entièrement relative à un sujet pensant. La volonté essentielle est une sorte de pulsion anonyme, une force vitale immanente à l'activité; elle produit une compréhension qui exprime son unité sans la médiation d'un projet, d'une représentation. La volonté arbitraire caractérise l'agent économique égoïste et dissimulateur et d'abord plus fondamentalement l'homme, c'est-à-dire l'être masculin (Tönnies, 1944, p. 139), tandis que la femme - du moins la femme jeune, car une femme vieille devient une sorte d'homme, alors que l'homme n'est à l'inverse pleinement masculin qu'avec l'âge (ibid., p. 145) - incarne la volonté essentielle. S'installant dans le chez soi, la femme pousse l'homme au dehors, tel est le mouvement essentiel qui amorce le passage de la communauté à la société partout dans la nature ${ }^{13}$.

${ }^{12}$ Sur l'influence de Schopenhauer chez les premiers sociologues, voir Mestrovic, 1989.

13 «Cependant, non seulement chez les hommes, mais au moins aussi chez les mammifères, et partout où la femelle doit consacrer une grande partie de son temps et de ses soins à la nichée, la vie du mâle est précisément à cause de cela plus active parce que c'est à lui qu'incombent les soins de la nourriture et de la lutte - en particulier l'attaque et le rapt - comme celle qui s'engage pour la possession de la femelle elle-même, l'obligeant à tuer ses rivaux. En tant que chasseur et bandit, il est stimulé à guetter et à écouter de loin» (Tönnies, 1944, p. 139). 
Cette distinction entre volonté vitale essentielle et volonté rationnelle arbitraire a pour conséquence la destitution du concept juridique de personne. En effet, la personne est, de l'aveu de tous, le suppôt de la volonté rationnelle et certainement pas de la volonté vitale, qui est anonyme et présente chez les non-personnes. Tönnies va cependant plus loin et, dans une sorte de mélange de la critique humienne de la substantialité du moi et de l'idée kantienne du je transcendantal, prétend que la personne est une fiction requise par nos croyances causales ou encore le corrélat idéal de la représentation ${ }^{14}$. Le sujet de la volonté essentielle est au contraire une réalité, précisément parce que ce n'est pas un suppôt «subjectif», mais un fondement organique. Les conséquences d'une telle conception sont considérables : elle tend à discréditer la délibération comme processus de décision et aussi le parlementarisme ou plus généralement le système de représentation des personnes. C'est la société en tant que rapport entre les volontés rationnelles arbitraires qui est elle-même entièrement relative au projet de ces volontés. De ce point de vue - détournement du concept hobbesien de l'autorisation —, «une société est un être imaginaire ou constitué par la pensée pour exprimer la volonté réfléchie commune de ses auteurs» (Tönnies, 1944, p. 216).

Pour Collingwood, seul le type de volonté que Tönnies calomnie sous le nom de volonté arbitraire est proprement volonté, c'est-à-dire capacité de décision. C'est cette décision pratique qui constitue ce que la théorie politique classique essaie de décrire comme un «contrat social» (NL 20.63); un bon exemple de cette décision pratique est donné en 20.91 : «la société est le fait que certaines personnes partagent une conscience sociale pratique exprimée verbalement en une formulation commune: "nous voulons faire cette promenade" ou "nous voulons monter ce bateau"». L'homme de la société est la personne juridique qui consiste essentiellement en une volonté. On ne doit pas surtout pas confondre le contrat social (au risque — ou dans l'intention — de le discréditer) avec l'échange marchand : le lien politique ne repose ni sur le devoir moral, ni sur l'utilité ou l'économie, mais sur une raison politique (voir «Political Action», dans Collingwood, 1989, p. 95), sur la perspective d'un bien proprement politique - essentiellement l'institution d'un ordre collectif qui favorise l'intérêt commun d'agents libres.

Les diagnostics sur le procès de civilisation sont alors inverses. Pour Tönnies, «l'ère de la société suit celle de la communauté» (Tönnies, 1944, p. 236), mais il faudrait dépasser le dépassement de la communauté. Il y a un mouvement qui part du communisme rural vers l'individualisme ruralurbain, puis vers l'individualisme indépendant dans la grande ville, puis le socialisme politique, et

14 «La personne ou le sujet de la volonté réfléchie est, comme toute formation de cette même volonté, unité par détermination extérieure, unum per accidens, unité mécanique. Plus particulièrement, tout comme la volonté de la personne n'a de réalité et d'unité que pour son sujet et par rapport à des actions possibles, de même le concept de la personne est une fiction ou (pensée comme réalité) une construction de la pensée scientifique destinée à exprimer l'unité de cause des formations de la volonté réfléchie» (Tönnies, 1944, p. 166). 
ensuite cosmopolitique (ibid., p. 240-241), dont la dissolution laisse peut-être espérer le retour du communisme. La politique de Collingwood est contraire. S'il y a toujours une trace de communauté dans la société ( $N L$ 21.5), il faut s'efforcer de la réduire, mais sans tomber dans l'illusion d'une société pure, d'une société universelle réalisée. Il y a une dimension dialectique à l'intérieur de toute communauté humaine, même la plus sociale; il y a déjà du social dans la communauté familiale, et encore du communautaire non social dans la société la plus avancée, comme le montre l'exemple de la société de mathématiques qui est volontairement une société de mathématiciens et involontairement une société d'anglophones (ibid., 21.52). Durkheim écrit contre Tönnies que «si la société est un fait de nature à son origine, elle reste telle jusqu'au terme de sa carrière» (Durkheim, 1975). Pour reconstituer la thèse de Collingwood, il faut modifier ainsi la formule : «si la société est un fait de volonté à sa fin, elle était telle dès le début de sa carrière». La «société universelle» ou cosmopolitique n'est pas une forme décadente mais la perspective, l'idéal vers quoi tend la société, sans jamais l'atteindre (NL 21.51). La solution de ce problème ne peut pas être définitive en raison du phénomène des générations, c'est-à-dire de «l'arrivage de nouveaux nourrissons dans le corps politique» (25.34).

On peut poursuivre la confrontation en notant que la différence entre les types, communauté et société, ne doit pas être faite selon les lignes que propose Tönnies; surtout, la priorité chronologique de la communauté sur la société n'entraîne pas une priorité en dignité et en valeur. Les termes se distribuent sur une échelle des formes qui exclut la position de types purs. Collingwood souligne que toute société concrète passe par une évolution de la communauté non sociale à la communauté sociale (ou société au sens strict), mais cette évolution n'est jamais dépassement complet du non social (21.5 : «aucune société réelle ne peut perdre toute trace de la communauté non sociale dont elle est sortie» voir aussi 37.28). Inversement, la communauté non sociale initiale n'est pas purement ou totalement non sociale, comme on le voit avec l'analyse de la famille ${ }^{15}$. L'opposition entre des types purs, à laquelle procède Tönnies, est éristique. La progression des espèces sur une échelle des formes est dialectique ${ }^{16}$. Cela dit, l'intention de Tönnies était de situer des concepts ou catégories pures, et non de

${ }^{15}$ Collingwood concède en apparence l'antériorité logique et (le plus souvent) chronologique de la communauté par rapport à la société ( $N L 20.31$ et 20.32). Mais en dépit de cela, il soutient très clairement, par exemple en 25.12, que le processus social est engagé dès les premières formes de la communauté : «À une étape qui se produit relativement tôt dans ce processus [dialectique] (il n’y a pas d'étape où il n'ait pas commencé à fonctionner) le corps politique est une communauté mixte qui consiste en un noyau social et un corps non social qui entoure le noyau. Les premiers sont appelés les gouvernants, les seconds les gouvernés».

${ }^{16}$ Selon le Nouveau Léviathan (33.81), l'éristique et la dialectique s'opposent comme le souci du combat et le souci de l'entente: «Dialectique [...] est le nom que Platon donne à une discussion paisible et amicale où les disputants cherchent un accord, et s'oppose à une discussion aigrie ou rendue belliqueuse parce qu'ils recherchent la victoire». 
décrire des formes historiques. La distinction entre communauté et société vaut au niveau de la philosophie sociale; au niveau de la sociologie empirique, il en va autrement puisqu'on a affaire non aux types purs mais à des mixtes. À cet égard, Tönnies et Collingwood semblent superficiellement d'accord. Superficiellement, car pour Tönnies c'est seulement dans l'empirie que la chose relève des deux types, tandis que dans la conception dialectique de Collingwood la mixité de la chose est aussi mobilité du concept.

Il conviendrait de mettre cette discussion en rapport avec ce que sont la civilisation et la barbarie selon Collingwood ( $N L$ 36.89, 36.9, 36.93 et 36.94). Pour Collingwood, une théorie du genre de celle de Tönnies est barbare. C'est en effet une théorie qui diminue la volonté libre et qui justifie les pratiques qui diminuent la volonté libre. Il y a ici dans la théorie «le sacrifice délibéré de la délibération» (13.55) ou encore «le projet de renoncer à la liberté, tant pratique, sous la forme d'une vie organisée, que théorique, sous la forme d'une vie scientifique, et d'agir ainsi de façon délibérée, en échangeant volontairement ce méprisable monde chrétien où nous sommes, contre un monde païen meilleur» (13.53). Car la volonté est capable de renoncer à elle-même et de se soumettre aux passions.

Est également intéressante cette idée que la société ne doit pas être pensée selon la tradition de l'économie politique, mais selon le modèle de ce que Collingwood appelle assez curieusement (car c'est une vision elliptique) le «droit romain». Collingwood fait appel à un autre Hegel que celui qui réfléchit sur la portée des transformations décrites par Adam Smith et Adam Ferguson. Non le Hegel de la vie éthique, pourtant si important pour le développement de l'idéalisme social et politique britannique, ni précisément le Hegel du «système des besoins», mais celui qui identifie, encore dans les Principes de la philosophie du droit, à la suite de Kant, personne et volonté et voit dans la famille une communauté déjà juridique, même si elle l'est «immédiatement». Ce rejet de la généalogie économique de la société civile, et plus généralement sa conviction que la raison de l'action proprement sociale n'est pas l'utilité, ni sa forme propre l'échange marchand, a conduit Collingwood à rester très allusif sur l'aspect économique de la justice sociale dont il affirme qu'elle conditionne le rapport entre les volontés libres. Le chapitre 38 porte sur cette question et se borne à affirmer que le contraste entre richesse et pauvreté dans une société n'est acceptable qu'à un degré qui conditionne le maintien du niveau de vie général; sinon ce contraste doit être diminué.

\section{Une autre sociologie ou pas de sociologie du tout?}

Le second objectif de cette étude est d'évaluer la portée de la polémique. Avons-nous affaire ici à une sociologie pure alternative ou bien à une destruction de la sociologie pure? Un premier élément de réponse tient au statut des concepts et de leur distinction. La vision qu'a Collingwood de la distinction selon une échelle des formes lui interdit autant de poser une seule différence de degré qu'une simple différence de nature (comme le fait Tönnies) entre communauté et société. En 1889, Durkheim, dans sa recension de Tönnies, bien avant la parution de son ouvrage De la division du travail social, accepte la description de chacun des termes de la distinction, et la thèse de la priorité de la 
communauté, mais reproche à l'Allemand de ne pas avoir vu la différence de degré : selon Durkheim, la société n'est pas plus naturelle que la communauté, son essence est fondamentalement la même, il n'y a pas de différence de nature; la recension suggère en outre que Tönnies aurait pu inverser son usage des termes «organique» et «mécanique» (Durkheim, 1975). En 1893, Tönnies prend sa revanche en donnant une recension de l'ouvrage de Durkheim sur la division du travail social (Aldous, 1972). Collingwood, quant à lui, ne saurait s'accorder avec Durkheim qui maintient la conception évolutionniste, la primauté de la pression du groupe, et tend à faire d'une certaine manière de la communauté le genre dont la société est seulement une forme moderne. Ces discussions traversent tout le $\mathrm{XIX}^{\mathrm{e}}$ siècle et elles sont, par exemple, au cœur de l'ouvrage de Jean Piaget, Le jugement moral chez l'enfant (1932), qui réintroduit contre Durkheim une différence de nature et fait de la société, et non de la communauté, le standard auquel il convient de confronter les organisations humaines.

Un deuxième point concerne la méthode. Remarquons que si Collingwood était une sorte de sociologue, il serait manifestement du côté de l'individualisme méthodologique, en dépit du fait qu'on associe usuellement les tendances dites idéalistes avec des conceptions holistes de la société. L’individualisme méthodologique est évident dans la conception de Collingwood.

Si une société consiste dans la conscience sociale pratique de ses membres, il s'ensuit qu'une société n'est rien de plus que ses membres. Elle n'a pas de volonté sauf la volonté de ses membres, pas d'activité sauf l'activité de ses membres, pas de responsabilité sauf la responsabilité de ses membres ( $N L$ 21.27).

On ne saurait dire plus clairement que les phénomènes sociaux sont le produit de l'agrégation d'actions individuelles. Attribuer de la volonté, de l'activité ou de la responsabilité à la société en tant que telle, c'est une forme de superstition qui repose sur une erreur à propos de la nature de la société. Dans «Political Action», Collingwood parle de «mythologie» à ce propos et rétorque que «la société n'est rien d'autre que les gens qu'elle contient» («Political Action», dans Collingwood, 1989, p. 102$103)$.

Mais l'individualisme méthodologique n'interdit pas, contrairement à ce qu'un regard superficiel pourrait faire croire, de prendre en compte les contextes, les relations et les structures sociales. L'explication par l'intériorisation des normes et de la pression du groupe subsiste localement dans la théorie de Collingwood, d'abord parce qu'elle convient partiellement à une dimension irréductible de la communauté, par laquelle celle-ci reste toujours sous un certain aspect non sociale et est régie par la contrainte - qui se situe, semble-t-il, sur la même échelle des formes que la liberté (NL 23.46), alors qu'il est dit ailleurs qu'autorité et force sont «entièrement» différentes (ibid., 21.72). Mais, même sous l'aspect social de la communauté, les acteurs capables de relations contractuelles s'insèrent dans des structures sociales qui sont données à l'avance et qui constituent le contexte de leurs actions. En particulier, le phénomène des générations, essentiel à la vie des sociétés, combine les situations d'autorité et les situations de liberté. Selon Collingwood, parmi les conditions de l'exercice 
d'une autorité (distincte de la force), il y a l'intelligence et la liberté de la part de celui qui est soumis à l'autorité (ibid., 21.71-72; voir aussi Collingwood, 1989, p. 103). Selon NL 21.76 et 21.13, la liberté de l'individu suppose l'intégration dans un contexte de liberté. Il existe des relations proprement sociales, au-delà des opérations individuelles; c'est d'ailleurs ce qui distingue de l'échange économique proprement dit la poursuite (individuelle) de l'utilité (ibid., 15.16).

Bref, la société n'est pas seulement produite par les volontés; elle constitue aussi leur contexte. Le paragraphe 21.19, qui fait de l'idée de soi un corrélat de l'idée d'un autre soi, est très instructif :

Nul n'a aucune idée de lui-même comme agent libre sans une idée d'agents libres autres que lui-même, et de relations sociales entre eux. Nul n'a une idée exacte de lui-même comme agent libre sans une idée exacte d'agents libres autres que lui-même et de relations sociales entre eux. (ibid., 21.19)

L'esprit consiste essentiellement en une volonté individuelle; la conscience de ma volonté est fonction de ma capacité à me distinguer de la volonté d'autrui (ibid., 21.1-14). Une conséquence politique de cette thèse de la priorité épistémique de la liberté d'autrui est que toute volonté cultive sa liberté en promouvant celle des autres, c'est-à-dire en promouvant l'égalité entre sa liberté et celle des autres :

L'idée de moi comme agent libre est inséparable de son corrélatif, l'idée d'agents libres autres que moi. Nul ne peut se penser libre si ce n'est comme intégré dans un contexte d'autres hommes libres constituant avec une lui une société. (ibid., 21.76)

Ou, comme il est dit dans «Political Action»: «Je ne peux organiser ma propre vie sans référence à l'organisation des autres» (Collingwood, 1989, p. 104). Bref, il ne faut surtout pas confondre cet individualisme méthodologique avec un solipsisme ou une thèse niant l'importance des contextes sociaux de l'action.

Mais le fait que les décisions des acteurs ont pour contexte les relations sociales n'empêche pas du tout que c'est la société qui a son principe dans la volonté, et non l'inverse. Cette position-ci manifeste une défiance de principe à l'égard des sciences sociales. C'est à partir de ce point que la philosophie sociale de Collingwood diverge d'une sociologie pure.

Par son individualisme et surtout par son usage dynamique des concepts de communauté et de société, Collingwood me semble moins éloigné de Max Weber que de Durkheim ou de Tönnies. Il suffit de rappeler que dans la première partie de son ouvrage posthume, Économie et société (1922), qui porte sur les catégories pures de la sociologie, Weber avait adapté la distinction entre communauté et société en la considérant comme une distinction entre des modes d'interaction entre les individus, et non pas comme une distinction entre des types de groupes, c'est-à-dire des entités indépendantes de ou antérieures à l'interaction. La substitution wébérienne des processus de Vergemeinschaftung et de Vergesellschaftung aux catégories statiques de Gemeinschaft et de Gesellschaft donne à cette 
opposition un style bien différent de la métaphysique de Tönnies et qui pourrait sembler compatible avec les grands traits d'une philosophie sociale collingwoodienne.

Cela dit, je doute qu'on trouve dans l'œuvre de Collingwood une conception de la rationalité pratique qui permette de développer une sociologie, même dans le style individualiste, pour la simple raison que la conception qu'a Collingwood du choix rationnel est éloignée des vues des économistes et des sociologues. Ici encore, comme c'est une échelle des formes qui rend compte des divers aspects de la rationalité pratique, une théorie unifiée, ou réductrice, de la décision n'est pas possible. Il faut se reporter aux indications que Collingwood donne sur les différences entre le «caprice» (choix sans raison) et les choix avec raison, c'est-à-dire les formes de la raison pratique que sont l'utilité, la règle, et le devoir, qui diffèrent en nature comme en degré (voir Connelly, 2007). Pour Collingwood, la bonne théorie de la décision est différenciée et ne saurait réduire la rationalité des acteurs aux comportements que l'on décrit en termes d'utilité ou même de régularité, et certainement pas de préférence arbitraire (le caprice est le choix irrationnel par excellence), pas plus qu'elle ne pourrait faire de la forme de la décision la plus haute sur l'échelle, l'agir par devoir, qui réalise le mieux l'essence du choix rationnel, un modèle explicatif pour tout comportement. Elle se doit, au contraire, de tenir compte de cette diversité et de cette gradation des formes de la raison pratique. L'essai «Political Action» insiste sur le fait que la politique ne diffère pas moins que la morale de l'économie (Collingwood, 1989, p. 94-95). Le rejet des modèles économiques de l'interaction sociale et de la raison pratique est aussi profond, chez Collingwood, que sa réserve à l'égard de la sociologie ${ }^{17}$.

\section{Bibliographie}

Adair-Toteff, C.

1995 «erdinand Tönnies: Utopian Visionary», Sociological Theory, vol. 13, nº 1, p. 58-65. Aldous, $\mathrm{J}$.

1972 «An Exchange Between Durkheim and Tonnies on the Nature of Social Relations», The American Journal of Sociology, vol. 77, $\mathrm{n}^{\circ}$ 6, p. 1191-1200.

Bond, $\mathrm{N}$.

2002 «Le refus de la bürgerliche Gesellschaft et la genèse de la sociologie moderne allemande : l'exemple de Gemeinschaft und Gesellschaft de Ferdinand Tönnies», dans C. ColliotThélène et J.-F. Kervégan, De la société à la sociologie, Lyon, ENS éditions, p. 93-120.

${ }^{17}$ Cet article est issu d'une communication présentée au colloque «Collingwood et la philosophie du vingtième siècle», organisé en octobre 2007 par l'Université du Québec à Montréal et l'Université de Montréal avec le soutien de la Collingwood Society, de la Political Studies Association et du Conseil de recherche en science humaine du Canada. 
Breuer, S.

2002 «De Tönnies à Weber. Sur l'existence d'un "courant allemand” en sociologie», dans C. Colliot-Thélène et J.-F. Kervégan, De la société à la sociologie, Lyon, ENS éditions, p. 121-147.

Collingwood, R. G.

1989 Essays in Political Philosophy, Oxford, Clarendon Press.

2001 Le Nouveau Léviathan, trad. par L. Carrive, Paris, Kimé.

Connelly, J.

2007 «Character, Duty and Historical Consciousness», dans W. Sweet, British Idealism: Moral, Social and Political Philosophy, Exeter, Imprint Academic.

Durkheim, E.

1975 «Communauté et société selon Tönnies (1889)», dans E. Durkheim, Textes 1, Paris, Minuit, p. 383-390.

Maine, H. S.

1861 Ancient Law. Its Connection With the Early History of Society, and Its Relations to Modern Ideas, Londres, Murray.

Mestrovic, S.

1989 «Rethinking the Will and Idea of Sociology in the Light of Schopenhauer's Philosophy», The British Journal of Sociology, vol. 40, $\mathrm{n}^{\mathrm{o}}$ 2, p. 271-293.

Mitzman, A.

1971 «Tönnies and German Society, 1887-1914: From Cultural Pessimism to Celebration of the Volksgemeinschaft», Journal of the History of Ideas, vol. 32, nº 4, p. 507-524.

Morgan, L. H.

1877 Ancient Society or Researches in the Lines of Human Progress from Savagery through Barbarism to Civilization, Londres, MacMillan Press.

Orenstein, $\mathrm{H}$.

1968 «The Ethnological Theories of Henry Sumner Maine», American Anthropologist, vol. 70, $\mathrm{n}^{\mathrm{o}} 2$, p. 264-276.

Sen, A.

2000 Repenser l'inégalité, trad. par P. Chemla, Paris, Seuil.

Tönnies, F.

1940 Fundamental Concepts of Sociology, trad. par Ch. Loomis, New York, American Book Company.

1944 Communauté et société. Catégories fondamentales de la sociologie pure, trad. de la $8^{\mathrm{e}}$ édition par J. Leif, Paris, Presses universitaires de France. 
Vance, R. B.

1941 «(Review) Fundamental Concepts of Sociology (Gemeinschaft und Gesellschaft) by Ferdinand Tönnies», Social Forces, vol. 20, nº 2, p. 272-273.

Weber, M.

2003 Économie et société, trad. par J. Freund, P. Kamnitzer, P. Bertrand, et al., Paris, Pocket. Wirth, L.

1926 «The Sociology of Ferdinand Tönnies», The American Journal of Sociology, vol. 32, $\mathrm{n}^{\circ} 3$, p. $412-422$. 